\title{
Publisher Correction: Zeb2 drives invasive and microbiota-dependent colon carcinoma
}

Karolina Slowicka, loanna Petta, Gillian Blancke, Esther Hoste (D), Emilie Dumas, Mozes Sze, Hanna Vikkula, Enrico Radaelli @, Jody J. Haigh, Sven Jonckheere, Joachim Taminau, Niels Vandamme, Andy Wullaert, Eugene Tulchinsky, David Nittner, Pieter Van Vlierberghe (D), Gert De Hertogh, Pamela Baldin, Emre Etlioglu (D, Pratyaksha Wirapati@, Louis Boon, Bart N. Lambrecht, Chris Callewaert, Sabine Tejpar, Steven Goossens, Geert Berx (1), Lars Vereecke and Geert van Loo (1)

Correction to: Nature Cancer https://doi.org/10.1038/s43018-020-0070-2, published online 15 June 2020.

In the version of this article initially published, affiliations for several authors (David Nittner, Pieter Van Vlierberghe, Gert De Hertogh, Pamela Baldin, Emre Etlioglu, Sabine Tejpar, Steven Goossens and Lars Vereecke) were incorrect or missing. The correct authors and affiliations are as follows:

Karolina Slowickaa ${ }^{1,2,3,21}$, Ioanna Petta ${ }^{1,3,4,21}$, Gillian Blancke ${ }^{1,3,4}$, Esther Hoste ${ }^{1,2}$, Emilie Dumas ${ }^{1,3,4}$, Mozes Sze $^{1,2}$, Hanna Vikkula ${ }^{1,2}$, Enrico

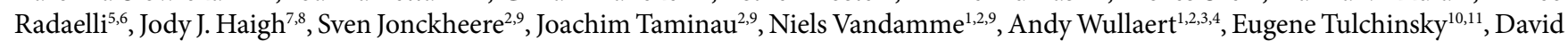
Nittner ${ }^{12,13}$, Pieter Van Vlierberghe ${ }^{9,14}$, Gert De Hertogh ${ }^{15}$, Pamela Baldin ${ }^{16}$, Emre Etlioglu ${ }^{13}$, Pratyaksha Wirapati ${ }^{17}$, Louis Boon ${ }^{18}$, Bart N. Lambrecht ${ }^{1,4}$, Chris Callewaert ${ }^{19}$, Sabine Tejpar ${ }^{13}$, Steven Goossens ${ }^{2,9,14,20}$, Geert Berx ${ }^{2,9,22}$, Lars Vereecke ${ }^{1,3,4,9,22}$ and Geert van Loo ${ }^{1,2,3,9,22}$

${ }^{1}$ VIB Center for Inflammation Research, Ghent, Belgium. ${ }^{2}$ Department of Biomedical Molecular Biology, Ghent University, Ghent, Belgium. ${ }^{3}$ Ghent Gut Inflammation Group, Ghent University, Ghent, Belgium. ${ }^{4}$ Department of Internal Medicine and Pediatrics, Ghent University, Ghent, Belgium. ${ }^{5} \mathrm{KU}$ Leuven Center for Human Genetics, Leuven, Belgium. ${ }^{6}$ VIB Center for Brain \& Disease Research, Leuven, Belgium. ${ }^{7}$ Research Institute in Oncology and Hematology, CancerCare Manitoba, Winnipeg, Manitoba, Canada. ${ }^{8}$ Department of Pharmacology and Therapeutics, Rady Faculty of Health Sciences, University of Manitoba, Winnipeg, Manitoba, Canada. ${ }^{9}$ Cancer Research Institute Ghent, Ghent, Belgium. ${ }^{10}$ University of Leicester, Leicester, UK. ${ }^{11}$ Department of Biomedical Sciences, Nazarbayev University School of Medicine, Astana, Kazakhstan. ${ }^{12}$ Histopathology Expertise Center, VIB-KU Leuven Center for Cancer Biology, Leuven, Belgium. ${ }^{13}$ Department of Oncology, University Hospital Leuven, KU Leuven, Leuven, Belgium. ${ }^{14}$ Department of Biomolecular Medicine, Ghent University, Ghent, Belgium. ${ }^{15}$ Department of Pathology, University Hospital Leuven, KU Leuven, Leuven, Belgium. ${ }^{16}$ Department of Pathology, Cliniques Universitaires Saint Luc, Université Catholique de Louvain, Brussels, Belgium. ${ }^{17}$ Swiss Institute of Bioinformatics, Lausanne, Switzerland. ${ }^{18}$ Bioceros, Utrecht, the Netherlands. ${ }^{19}$ Center for Microbial Ecology and Technology, Ghent University, Ghent, Belgium. ${ }^{20}$ Department of Diagnostic Sciences, Ghent University, Ghent, Belgium. ${ }^{21}$ These authors contributed equally: Karolina Slowicka, Ioanna Petta. ${ }^{22}$ These authors jointly supervised this work: Geert Berx, Lars Vereecke, Geert van Loo.

The errors have been corrected in the HTML and PDF versions of the article.

Published online: 10 July 2020

https://doi.org/10.1038/s43018-020-0097-4

(c) The Author(s), under exclusive licence to Springer Nature America, Inc. 2020 